\title{
Angiopoietin-I inhibits tumour growth and ascites formation in a murine model of peritoneal carcinomatosis
}

\author{
O Stoeltzing', SA Ahmad', W Liu', MF McCarty', AA Parikh², F Fan', N Reinmuth', CD Bucana' and \\ LM Ellis*,1,2
}

'Department of Cancer Biology, The University of Texas M.D. Anderson Cancer Center, Houston, Texas, TX 77030, USA; '2Department of Surgical Oncology, The University of Texas M.D. Anderson Cancer Center, Houston, Texas, TX 77030, USA

Angiopoietin-I is an important regulator of endothelial cell survival. Angiopoietin-I also reduces vascular permeability mediated by vascular endothelial growth factor. The effects of angiopoietin-I on tumour growth and angiogenesis are controversial. We hypothesised that angiopoietin-I would decrease tumour growth and ascites formation in peritoneal carcinomatosis. Human colon cancer cells (KMI2L4) were transfected with vector ( $p c D N A)$ alone (control) or vector containing angiopoietin-I and injected into the peritoneal cavities of mice. After 30 days, the following parameters were measured: number of peritoneal nodules, ascites volume, and diameter of the largest tumour. Effects of angiopoietin-I on vascular permeability were investigated using an intradermal Miles assay with conditioned media from transfected cells. Seven of the nine mice in the pcDNA group developed ascites $(1.3 \pm 0.5 \mathrm{ml}$ (mean \pm s.e.m.)), whereas no ascites was detectable in the angiopoietin-I group $(0$ out of 10$)(P<0.0$ I). Number of peritoneal metastases $(P<0.05)$, tumour volume, $(P<0.05)$, vessel counts $(P<0.0 \mathrm{I})$, and tumour cell proliferation $(P<0.0 \mathrm{I})$ were significantly reduced in angiopoietin-I-expressing tumours. Conditioned medium from angiopoietin-I-transfected cells decreased vascular permeability more than did conditioned medium from control cells $(P<0.05)$. Our results suggest that angiopoietin-I is an important mediator of angiogenesis and vascular permeability and thus could theoretically serve as an anti-neoplastic agent for patients with carcinomatosis from colorectal cancer.

British Journal of Cancer (2002) 87, I| 82 - | | 87. doi: |0. I038/sj.bjc.6600598 www.bjcancer.com

(C) 2002 Cancer Research UK

Keywords: angiopoietins; angiogenesis; vascular permeability; ascites; colon cancer

Angiogenesis has been associated with aggressive disease in human colorectal cancer (Takahashi et al, 1995, 1998). Although the vascular endothelial growth factor (VEGF) receptor-ligand system has been implicated as a critical mediator of vasculogenesis and angiogenesis, the angiopoietins (Ang-1 to Ang-4) have also been shown to be important regulators of neovascularisation and endothelial cell survival in malignant and non-malignant tissues (Peters, 1998). Ang-1 has been recognised as the major ligand that activates the tyrosine kinase receptor Tie2, thereby promoting endothelial cell survival and vessel stabilisation by recruiting and sustaining peri-endothelial supporting cells (Fujikawa et al, 1999; Hayes et al, 1999; Kwak et al, 1999; Papapetropoulos et al, 1999). Ang-2 is the naturally occurring antagonist to Ang-1; Ang-2 prevents Tie2 activation and results in vessel destabilisation (Maisonpierre et al, 1997). Studies in transgenic mice revealed that inactivation of Ang-1 or the Tie2 receptor causes embryos to die from defects in vascular remodelling (Dumont et al, 1994; Sato et al, 1995; Suri et al, 1996). Recently, studies have shown that the angiopoietins are sequentially expressed during the angiogenic process, indicating that they are also important modulators of post-natal neovascularisation. Balanced and sequential expression of the angiopoietins and VEGF is required for successful angiogenesis (Asahara et al, 1998; Ray et al, 2000). In addition, Ang-1 has been

*Correspondence: Dr LM Ellis, Department of Surgical Oncology, Box 444, The University of Texas M. D. Anderson Cancer Center, 1515 Holcombe Blvd., Houston, TX 77030-4009, USA; E-mail: lellis@mdanderson.org Received 2 May 2002; revised 5 August 2002; accepted 19 August 2002 shown to override VEGF-mediated effects on vascular permeability (vessel leakage) of adult vasculature (Thurston et al, 1999, 2000).

The effects of the angiopoietins on angiogenesis, tumour growth and vascular permeability remain controversial (Suri et al, 1998; Chae et al, 2000; Hayes et al, 2000; Ahmad et al, 2001; Shim et $a l, 2001)$. Some studies suggest that Ang-1 may be pro-angiogenic (Suri et al, 1998; Hansbury et al, 2001; Shim et al, 2001), whereas others have shown that Ang-1 inhibits angiogenesis, tumour growth and vascular permeability (Chae et al, 2000; Hayes et al, 2000; Thurston et al, 2000; Ahmad et al, 2001; Tian et al, 2002).

We hypothesised that overexpression of Ang-1 by human colon cancer cells would decrease angiogenesis, tumour growth and metastasis formation and inhibit ascites formation in an experimental model of peritoneal carcinomatosis. Our results suggest that Ang1 is an important regulator of angiogenesis and vascular permeability and thus indicate that Ang-1 could theoretically serve as an antiangiogenic agent in the treatment of metastatic colorectal cancer.

\section{MATERIALS AND METHODS}

\section{Cell culture and transfection}

The highly metastatic human colon cancer cell line KM12L4 was obtained from IJ Fidler (The University of Texas M.D. Anderson Cancer Center) (Morikawa et al, 1988). KM12L4 cells were cultured and maintained on plastic in modified Eagle's medium supplemented with $10 \%$ foetal bovine serum, 2 units $\mathrm{ml}^{-1}$ penicillin-streptomycin mixture (Flow Laboratories, Rockville, MD, 
USA), two-fold vitamin solution (Life Technologies, Inc., Grand Island, NY, USA), $1 \mathrm{mM}$ sodium pyruvate, $2 \mathrm{mM}$ L-glutamine and non-essential amino acids and incubated in 5\% $\mathrm{CO}_{2}-95 \%$ air at $37^{\circ} \mathrm{C}$ as previously described (Shaheen et al, 2001).

The full-length cDNA for Ang-1 was provided by Tona Gilmer (GlaxoSmithKline, Research Triangle Park, NC, USA). The construct was sub-cloned into a pcDNA3 vector (InVitrogen, Carlsbad, CA, USA) containing a hygromycin resistance gene (Ahmad et al, 2001). Vector alone or vector containing Ang-1 was transfected into KM12L4 cells using Lipofectin according to the manufacturer's protocol (Boehringer Mannheim Co., Randburg, South Africa), and the transfected cells were grown in selective media as previously described (Ahmad et al, 2001). Successful transfection of Ang-1 was confirmed by Northern blot analysis as previously described (Ahmad et al, 2001).

For in vivo studies, Ang-1- and pcDNA-transfected KM12L4 cells were harvested from sub-confluent cultures. Briefly, cells were rinsed with phosphate-buffered saline (PBS), trypsinised $(0.25 \%$ trypsin; $0.02 \%$ EDTA) and re-suspended in $10 \%$ foetal bovine serum-modified Eagle's medium. Cells were counted and cell viability was assessed using the Trypan blue dye exclusion assay ( $>90 \%$ viability). Cells were resuspended in Hank's balanced salt solution for tumour cell inoculation into mice.

\section{In vivo studies}

Eight-week-old male athymic nude mice (obtained from the Animal Production Area of the National Cancer Institute and Development Center, Frederick, MD, USA) were acclimated for 1-2 weeks while caged in groups of five. Mice were housed as previously described (Shaheen et al, 2001) and fed a diet of animal chow and water ad libitum throughout the experiment. Experiments were approved by the Animal Care and Use Committee at M. D. Anderson Cancer Center and met all the standards required by the UKCCCR guidelines for the welfare of animals in experimental neoplasia, as published (United Kingdom Co-Ordinating Committee on Cancer Research, 1998).

Peritoneal carcinomatosis model Mice were randomly assigned to one of two groups ( $n=10$ per group). The average body weight among groups was similar at assignment. Under sterile conditions, either Ang-1- or pcDNA (control)-transfected KM12L4 cells $\left(1 \times 10^{6}\right.$ cells) were injected into the peritoneal cavity of each mouse by means of a 30-gauge needle. An injection volume of $500 \mu$ l (in Hank's balanced salt solution) was used to achieve a distribution adequate for peritoneal implantation of tumour cells. Animals were monitored daily (weighed once per week), and when mice in any group showed early signs of decreased mobility, all animals were killed by cervical dislocation after anaesthesia induction with pentobarbital (Nembutal, $50 \mathrm{mg} \mathrm{kg}^{-1}$ ). Body weight was determined, and ascites was collected by performing a lower midline incision and completely draining the intra-abdominal fluid into a flask. The ascites volume was then measured with a 1-ml tuberculin syringe. Incisions were then extended to allow photography of the peritoneal cavity and determination of the extent of peritoneal carcinomatosis. Peritoneal tumour nodules were counted, and the diameter of the largest tumour (always occurring in the greater omentum) was determined. Tumour tissue was then harvested, placed in either $10 \%$ formalin for paraffin embedding or snap-frozen in optimum cutting temperature solution (Miles Inc., Elkhart, IN, USA) in preparation for subsequent immunohistochemical analyses.

Intradermal Miles assay To investigate the effects of overexpression of Ang-1 by tumour cells on vascular permeability, the intradermal Miles assay was performed as described previously (Zebrowski et al, 1999). Conditioned medium (CM) from Ang-1and pcDNA-transfected KM12L4 cells was collected after a 48-h incubation in $5 \%$ foetal bovine serum-modified Eagle's medium at $80 \%$ cell density, centrifuged for $5 \mathrm{~min}$ at 2000 r.p.m. and filtered through a $0.22-\mu \mathrm{m}$ filter. Mice ( $n=3$ per group) were injected intravenously with sterile $0.5 \%$ Evans blue dye $(200 \mu \mathrm{l})$ via the tail vein. Ten minutes later, mice were given four separate intradermal injections at different sites into the dorsal skin - one each of CM-Ang-1, CM-pcDNA, PBS (negative control), and PBS plus VEGF $\left(10 \mathrm{ng} \mathrm{ml}^{-1}\right)$ (R\&D Systems Inc., Minneapolis, MN, USA) (positive control for increased vascular permeability). The injections were done using a 30-gauge needle, and the injection volume was $50 \mu$ l. Mice were sacrificed $20 \mathrm{~min}$ after intradermal injections by cervical dislocation following anaesthesia induction with nembutal. The dorsal skin of each mouse was harvested to permit visualisation of intradermal dye leakage. To determine the relative degree of vascular permeability, two dimensions ( $a$ and $b$ ) of the elliptically appearing area of dye leakage were obtained at each injection site and the area was calculated by the formula $a \times b \times \pi$. Densitometric analysis was performed using the NIH Image Analysis software (V1.62) from the National Institute of Health (Bethesda, MD, USA) as another means of quantifying the extent of dye leakage at the intradermal injection sites in each mouse. Digitally obtained images of the underside of the dorsal skin, including all injection sites, were converted to a gray-scale image, and dye density was analysed at each site (threshold was set individually for each dorsal skin flap, but was constant for each mouse).

\section{Immunohistochemical analyses of tumour vessel density}

Antibodies for immunohistochemical analyses were obtained as follows: rat anti-mouse CD31 antibody from PharMingen (San Diego, CA, USA) and peroxidase-conjugated goat anti-rat IgG from Jackson Research Laboratories (West Grove, PA, USA). Tumours that had been frozen in optimum cutting temperature solution were sectioned at $8-\mu \mathrm{m}$ intervals, mounted on positively charged slides and air-dried for $30 \mathrm{~min}$. Tissue sections were then fixed in cold acetone, followed by $1: 1$ acetone/chloroform and acetone, and then washed with PBS. Specimens were then incubated with $3 \% \mathrm{H}_{2} \mathrm{O}_{2}$ in methanol for $12 \mathrm{~min}$ at room temperature to block endogenous peroxidase, washed with $\mathrm{PBS}(\mathrm{pH} 7.5)$ and incubated for $20 \mathrm{~min}$ at room temperature in a protein-blocking solution consisting of PBS supplemented with $1 \%$ normal goat serum and $5 \%$ normal horse serum. The primary antibody directed against CD31 was diluted 1:800 in protein-blocking solution and applied to the sections, which were incubated overnight at $4^{\circ} \mathrm{C}$. Sections were then rinsed in PBS and incubated for $10 \mathrm{~min}$ in proteinblocking solution before the addition of peroxidase-conjugated secondary antibody. The secondary antibody used for CD31 staining (peroxidase-conjugated goat anti-rat IgG) was diluted $1: 200$ in protein-blocking solution. After incubation with the secondary antibody for $1 \mathrm{~h}$ at room temperature, the samples were washed and incubated with stable diaminobenzidine substrate (Research Genetics, Huntsville, AL, USA). Staining was monitored under a bright-field microscope, and the reaction was stopped by washing with distilled water. Sections were counterstained with Gill No. 3 haematoxylin solution (Sigma Chemical Co., St. Louis, MO, USA) and mounted with Universal Mount (Research Genetics). CD31-stained vessels were counted at $50 \times$ magnification at four different quadrants of each tumour $(2 \mathrm{~mm}$ inside the tumournormal tissue interface), and average vessel counts were calculated. For all immunohistochemical procedures, the primary antibody was omitted as a negative control.

\section{Immunohistochemical analyses of tumour cell proliferation}

Paraffin-embedded tumours were sectioned at 4- to 6- $\mu \mathrm{m}$ intervals, mounted on positively charged Superfrost slides (Fisher Scientific 
Co., Houston, TX, USA) and allowed to dry overnight at room temperature. Sections were deparaffinised in xylene, treated with a graded series of alcohol washes $(100,95$, and $80 \%$ ethanol in double-distilled $\mathrm{H}_{2} \mathrm{O}\left(\mathrm{v} \mathrm{v}^{-1}\right)$ ), rehydrated in PBS ( $\mathrm{pH}$ 7.5) and microwaved for $5 \mathrm{~min}$ for antigen retrieval. Tumour sections were stained for proliferating cell nuclear antigen (PCNA) using mouse anti-PCNA clone PC10 DAKO A/S (DAKO Corp., Carpinteria, CA, USA). Immunohistochemical procedures were performed as described previously (Ahmad et al, 2001). Positive reactions were visualised by incubating the slides with stable diaminobenzidine for 10-20 min. The sections were rinsed with distilled water, counterstained with Gill No. 3 haematoxylin solution for $1 \mathrm{~min}$, and mounted with Universal Mount. The number of tumour cells staining and not staining for PCNA was determined (at $100 \times$ magnification) at four staining 'hot spots' per tumour sample, and the percentage of PCNA-positive cells was then calculated. Necrotic areas of the tumour were excluded. Slides were also stained with haematoxylin and eosin to permit study of overall tissue morphology.

\section{Statistical analyses}

All statistical analyses were done using InStat Statistical Software version 2.03 (GraphPad Software, San Diego, CA, USA), with $P$ values less than 0.05 considered to be statistically significant. Tumour-associated parameters were tested for statistical significance using the two-sided Student's $t$-test or Mann-Whitney $U$-test (for non-parametric data). Fisher's exact test was applied for comparing incidences of peritoneal carcinomatosis and ascites formation.

\section{RESULTS}

\section{Effect of angiopoietin-1 expression on formation of peritoneal metastases and tumour growth}

To study the effect of Ang-1 on peritoneal implantation and growth of human colon cancer cells, pcDNA- and Ang-1-transfected KM12L4 cells were injected into the peritoneal cavities of nude mice. Thirty days after tumour cell inoculation, three of the control (pcDNA) mice showed decreased mobility (secondary to ascites), and the experiment was terminated. (One mouse in the pcDNA group died at day 21 of acute bowel obstruction caused by a peritoneal tumour). Peritoneal carcinomatosis developed in seven $(78 \%)$ of the nine mice in the control group, compared to three $(30 \%)$ of the 10 mice of the Ang-1 group. However, the incidences of development of carcinomatosis were not significantly different $(P=0.07)$.

To evaluate the effects of Ang-1 overexpression on tumour burden in mice, the number of peritoneal metastases and the volume of the largest tumour (occurring in the greater omentum) were calculated for each mouse. The total number of peritoneal metastases was significantly lower in the Ang-1 group than in the control group $(P<0.01)$ (Figure 1A). Peritoneal nodules were additionally categorised into size ranges (greatest diameter), as a more precise volume measurement (cubic $\mathrm{mm}$ ) was not feasible due to their abundance. Small tumours $(1-5 \mathrm{~mm})$ were detectable at frequency of $2.2 \pm 1.4$ nodules/mouse (mean \pm s.e.m.)) in the pcDNA group and a mean of $0.3 \pm 0.2$ nodules/mouse in the Ang-1 group $(P<0.05)$. Large tumours $(6-15 \mathrm{~mm})$ were detectable at frequency of $13.4 \pm 3.3$ nodules/mouse in the pcDNA group and compared to $0.5 \pm 0.3$ nodules/mouse in the Ang-1 group $(0.5 \pm 0.3) \quad(P<0.05)$. As tumour growth beyond $1-2 \mathrm{~mm}$ is angiogenesis dependent, measurement of the largest tumour was a consistent and unbiased reflection of the effects of Ang-1 on tumour angiogenesis. The maximal tumour volume was significantly less in the Ang-1 group than in the pcDNA group $(P<0.05)$ (Figure 1B).

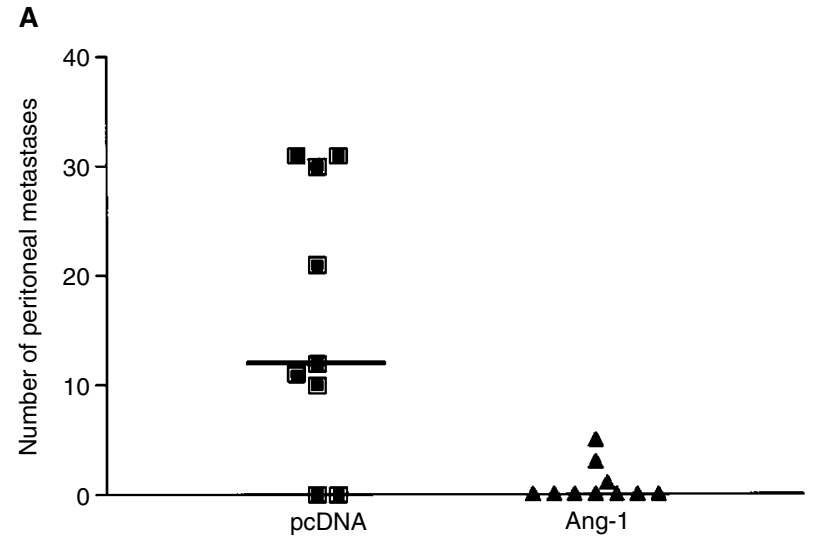

B

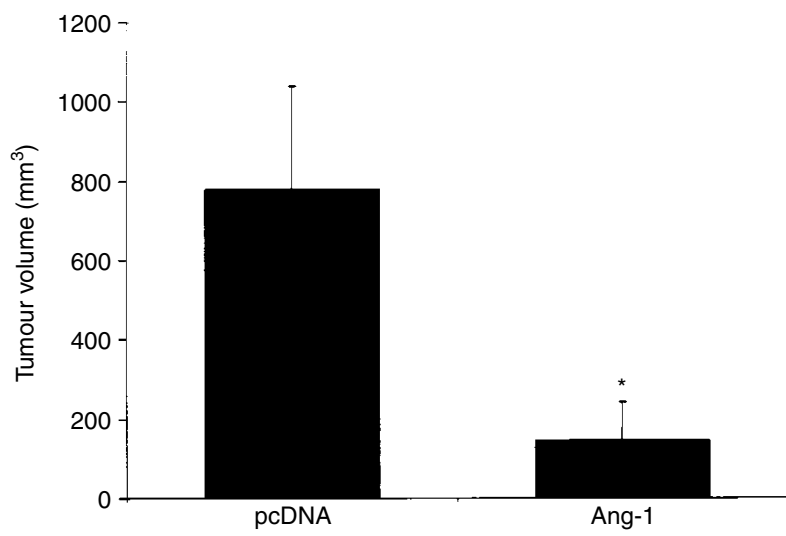

Figure I Effect of Ang-I overexpression on tumour growth. Ang-I- or pcDNA-transfected KMI2L4 colon cancer cells were injected into the peritoneal cavities of mice. (A) After 30 days, mice were killed and peritoneal metastases were counted. Overexpression of Ang-I in colon cancer cells significantly reduced the number of peritoneal metastases $(P<0.01$, Mann - Whitney U-test) compared to controls (pcDNA). Bars: median. (B) The largest tumour (greater omentum) was measured in each mouse. Tumour volumes in the Ang- I group were significantly smaller than tumours in the pcDNA group $(P<0.05$, Mann - Whitney $U$-test). Bars: s.e.m.

\section{Effect of angiopoietin-1 expression on ascites formation}

The incidence of ascites formation was significantly higher in mice injected with pcDNA-transfected KM12L4 cells (7 out of $9,78 \%)$ than in mice injected with Ang-1-overexpressing KM12L4 cells (0 out of 10$)(P<0.01$, Fisher's exact test). The ascites volumes in peritoneal metastases-bearing mice in the pcDNA group ranged from $0.3 \mathrm{ml}$ to $5 \mathrm{ml}$, with a median ascites volume of $1.3 \mathrm{ml}$ (haemorrhagic ascites in all cases). The median ascites volume was significantly higher in the pcDNA group $(P<0.01)$ than in the Ang-1 group as none of the Ang-1 mice developed ascites even though three of them had peritoneal tumours (Figure 2).

\section{Effect of angiopoietin-1 expression on microvessel density and tumour cell proliferation}

To determine the effects of Ang-1 overexpression on tumour neovascularisation and tumour cell proliferation, peritoneal metastases were sectioned and evaluated by immunohistochemical 
analyses. Microvessel density was significantly reduced in Ang-1transfected KM12L4 tumours relative to controls (pcDNA) $(P<0.01)$ (Figures $3 \mathrm{~A}$ and 4$)$. In addition, analysis of PCNA immunohistochemical staining revealed that Ang-1-overexpressing tumours had a significantly lower percentage of proliferating tumour cells in peritoneal metastases compared to control tumours $(P<0.01)$ (Figures 3B and 4$)$.

\section{Effect of angiopoietin-1 expression on vascular permeability}

To demonstrate whether the observed inhibition of ascites formation was attributable to reduced vascular permeability mediated by Ang-1 and not solely dependent on tumour burden, we used the Miles in vivo permeability assay to determine the direct effect of Ang-1 on vascular permeability. In this assay, effects on vascular permeability were investigated using conditioned media from Ang-1- or pcDNA-transfected KM12L4 cells. Injections with PBS and PBS plus VEGF served as a negative and positive control, respectively. Areas of intradermal dye leakage (a measure of vascular permeability) were significantly smaller at CM-Ang-1 injection sites than at CM-pcDNA injection sites (or PBS-plus-VEGF injection sites, the positive control) $(P<0.05)$ (Figure 5). All injection sites were additionally analysed by densitometry to verify these results. Dye density at CM-Ang-1 sites $\left(39.4 \pm 8.2\right.$ pixels $^{2} \times 10^{3}$ (mean \pm s.e.m. $)$ ) was significantly lower compared to CM-pcDNA sites $\left(76.8 \pm 2.9\right.$ pixels $\left.^{2} \times 10^{3}\right)(P<0.01$, Student's $t$-test $)$.

\section{DISCUSSION}

In the present study, overexpression of Ang-1 by human colon cancer cells inhibited tumour angiogenesis, growth of peritoneal metastases and ascites formation in an experimental model of peritoneal carcinomatosis. In studies using the HT29 human colon cancer cell line transfected with Ang-1, similar growth-inhibitory results were obtained (data not shown).

The effect of Ang-1 on tumour neovascularisation and growth is controversial. The concept that the angiopoietins are important mediators of tumour growth was elegantly demonstrated by Holash and associates, who demonstrated the importance of the coordinated induction of the angiopoietins and VEGF in tumour angiogenesis (Holash et al, 1999). Other studies have suggested that Ang-1 may be pro-angiogenic. Shim et al (2001) recently demonstrated that overexpression of antisense Ang-1 mRNA by HeLa cells inhibited angiogenesis and xenografted tumour growth in immunodeficient mice. An increased neovascularisation effect by Ang-1 overexpression was also described by Suri et al (1998) using a transgenic mouse model. These authors concluded that Ang-1 could be used in combination with VEGF to promote therapeutic angiogenesis.

The importance of the cooperation of Ang-1 and VEGF in induction of angiogenesis has been demonstrated in several malignant and non-malignant models of angiogenesis (Peters, 1998; Ray et al, 2000). In a transgenic mouse model with increased co-expression of Ang-1 and VEGF, angiogenesis was also increased (Thurston et al, 1999). This phenomenon of an enhanced vasculogenesis by combining Ang-1 with VEGF was also demonstrated by Chae et al (2000). Using a rabbit ischaemic hind-limb model, they demonstrated that a combination of Ang-1 and VEGF gene delivery resulted in the formation of larger blood vessels, increased blood flow and higher capillary density than was seen when either factor was delivered alone.

In contrast, several studies suggest that Ang-1 may inhibit tumour angiogenesis. In our previous studies, we were able to demonstrate that imbalances in Ang expression may regulate growth and angiogenesis of human colon cancer (Ahmad et al, 2001). In a subcutaneous xenografted colon cancer model, differen-

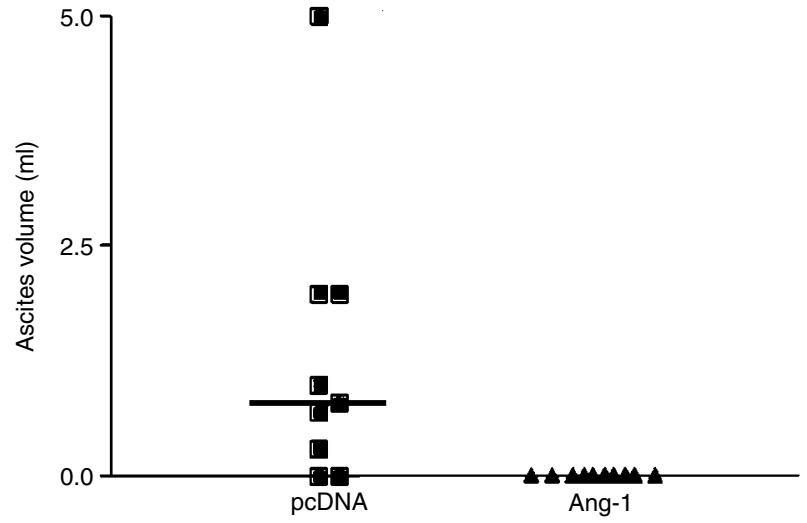

Figure 2 Impact of Ang-I expression on ascites formation in peritoneal carcinomatosis. Thirty days after tumour cell inoculation, ascites volumes were measured in each mouse. Seven of the nine mice in the pcDNA group developed detectable ascites, with a median ascites volume of $1.3 \mathrm{ml}$. In contrast, none of the 10 mice in the Ang-I group developed ascites $(P<0.01$, Fisher's Exact test). Bars: median.

A

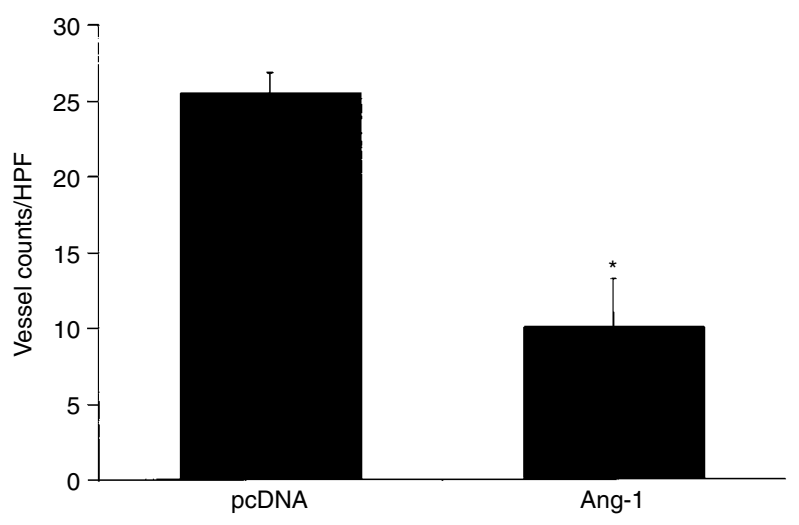

B

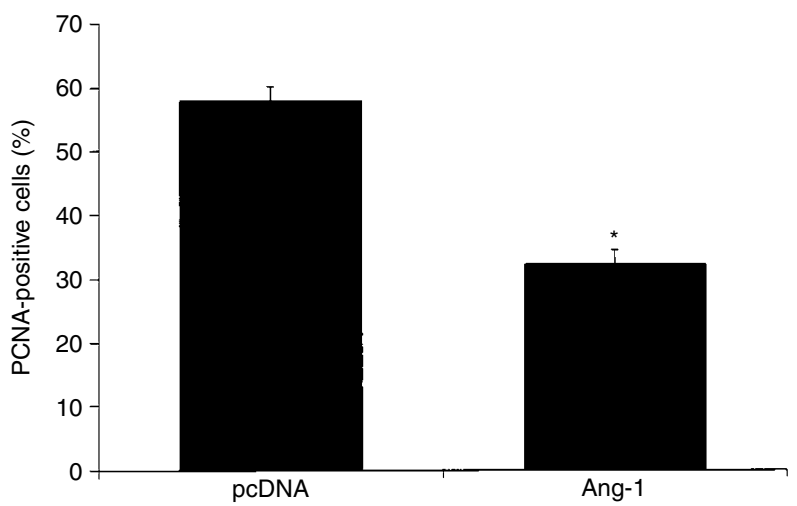

Figure 3 Effect of Ang-I overexpression on tumour vessel density and tumour cell proliferation. (A) Tumour vessels were stained for CD3I, and vessel counts were performed as described in Materials and Methods. Vessel counts in Ang-I tumours were significantly lower than vessel counts in pcDNA tumours ( ${ }^{*} P<0.01$, two-sided Student's t-test). (B) In addition, the percentage of proliferating tumour cells identified by PCNA staining was significantly reduced in Ang-I-overexpressing tumours $(* P<0.01$, two-sided Student's t-test). Bars: s.e.m. HPF, high-power field. 


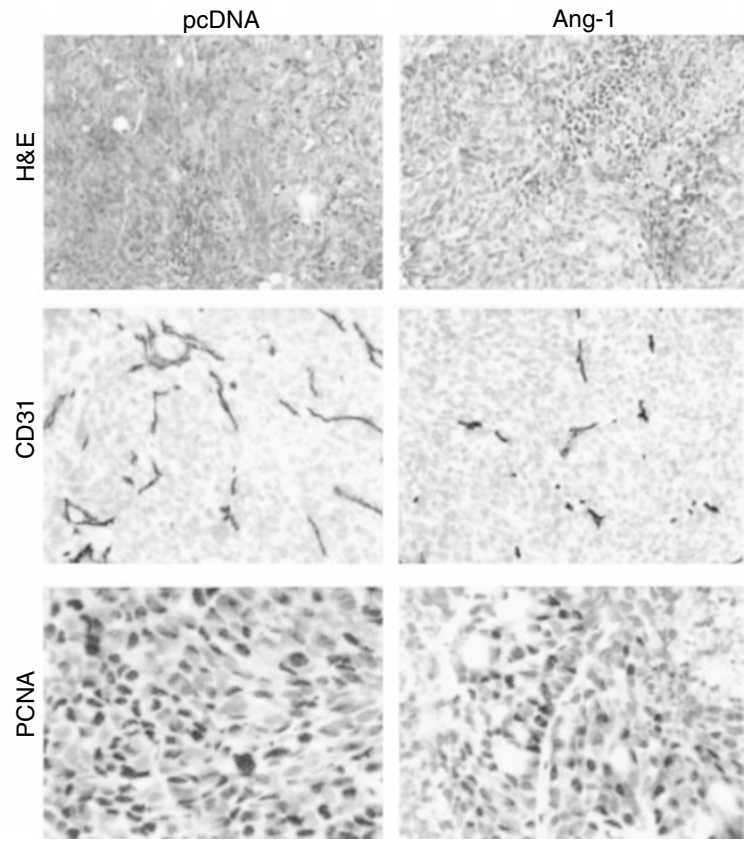

Figure 4 Immunohistochemical analysis of tumour vessel density (CD3I) and tumour cell proliferation (PCNA) in peritoneal tumours. Tumour sections were stained with haematoxylin and eosin (H\&E), anti-CD3I antibody or PCNA antibody as described in Materials and Methods. Images were obtained at $50 \times(\mathrm{H} \& \mathrm{E}, \mathrm{CD} 3 \mathrm{I})$ or $100 \times(\mathrm{PCNA})$ magnification.
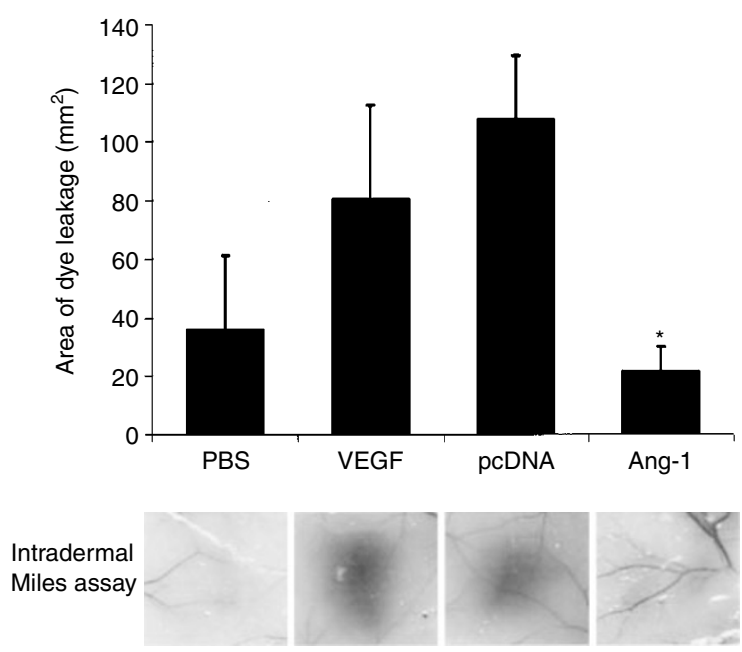

Figure 5 Effect of Ang-I overexpression by tumour cells on dermal vascular permeability. Effects on vascular permeability were examined using the Miles in vivo permeability assay. CM from Ang-I- or pcDNA-transfected cells was injected into the dermis of mice. After 20 min, mice were killed, and the areas of dye leakage (as a measure of permeability) were calculated for each injection site. PBS and PBS plus VEGF $\left(10 \mathrm{ng} \mathrm{ml^{-1 }}\right)$ served as negative and positive controls, respectively. In contrast to CM of pcDNA-transfected cells, Ang-I-containing CM did not increase plasma leakage, indicating that Ang-I abrogated permeability effects of tumour cell-derived growth factors in CM (*P $<0.05$, two-sided Student $t$-test). Bars: s.e.m.

tial expression of angiopoietins affected both tumour growth and angiogenesis. Ang-1 overexpression significantly inhibited tumour growth and angiogenesis in this model (Ahmad et al, 2001). These findings are now supported by the results of a recent study by Tian et al (2002), who found that Ang-1 overexpression by MCF-7 breast cancer cells resulted in stabilisation of tumour edge-associated blood vessels. In addition, tumour cell proliferation decreased significantly in the presence of Ang-1, resulting in a reduced xenografted tumour growth. Tie2 receptor was found to be present in vascular smooth muscle cells in culture in addition to endothelial cells. On the basis of these results, Tian et al (2002) concluded that vascular stabilisation by Ang-1 accounts for the inhibition of tumour growth. In a previous study, Hayes et al (2000) also demonstrated that Ang-1 overexpression in MCF-7 human breast cancer cells caused a significant retardation in tumour growth despite the high co-expression of a potent angiogenic growth factor (fibroblast growth factor-1).

The results of our present study suggest that the effects of Ang-1 on tumour growth were mediated by inhibition of tumour angiogenesis and that the abrogation of ascites formation was due to a reduction of vascular permeability (plasma leakage). In this model, Ang-1 overexpression did not prevent peritoneal implantation of tumours, although peritoneal nodules were fewer and smaller in the Ang-1 group. Therefore, Ang-1 prevented angiogenesis dependent tumour outgrowth by inhibiting neovascularisation, which was reflected by measurement of the largest tumour in mice.

As demonstrated in our permeability assay, Ang-1 levels in CM from Ang-1-transfected cells abrogated the increase of plasma leakage (dye leakage) of dermal microvasculature caused by tumour cell-derived growth factors (KM12L4 cells express relatively high amounts of VEGF (Ellis et al, 1996)), suggesting that Ang-1 is an important mediator of vascular stabilisation and permeability and may override VEGF-mediated vessel leakage. Other factors might have been present in conventional medium or conditioned medium that was used in this assay that potentially might have affected vascular permeability. However, VEGF is known as the strongest inducer of vascular leakage (Dvorak, 2000; Dvorak et al, 1999). Therefore, although possible, it is unlikely that ingredients of conventional medium and factors besides VEGF played a significant role in this assay. This hypothesis is supported by the results of other groups who have investigated the effects of Ang-1 on vascular permeability and vessel stabilisation. Gamble et al (2000) showed that administration of recombinant Ang-1 supported the localisation of a cell adhesion molecule (PECAM-1) into junctions between endothelial cells, strengthening these junctions. In addition, basal permeability of endothelial cell monolayers was reduced in a dose-dependent manner by treatment with recombinant Ang-1, and endothelial cell permeability responses to permeability-inducing agents such as thrombin and VEGF were inhibited by treatment with Ang-1. Thurston et al (1999) have reported anti-permeability properties of Ang-1 in two different studies evaluating the impact of VEGF on plasma leakage of adult vasculature and in a previous experiment using a transgenic mouse model overexpressing both Ang-1 and VEGF. In the latter study, co-expression of Ang-1 and VEGF had an additive effect on angiogenesis but resulted in the formation of leakage-resistant vessels. Furthermore, the authors showed that acute administration of Ang-1 protected adult vasculature from leakage mediated by VEGF and inflammatory cytokines (Thurston et al, 2000).

In conclusion, our study indicates that Ang-1 was an important mediator of tumour angiogenesis and vascular permeability in an experimental model of peritoneal carcinomatosis from human colon cancer. The inhibition of vascular permeability by Ang-1 is especially relevant in a tumour system associated with a great deal of morbidity due to ascites formation that accompanies carcinomatosis. Our studies suggest that Ang-1 could potentially serve as an anti-angiogenic/anti-permeability agent in the treatment of carcinomatosis from colorectal cancer. 


\section{ACKNOWLEDGEMENTS}

We thank Stephanie Deming from the Department of Scientific Publications and Rita Hernandez from the Department of Surgical Oncology at M. D. Anderson for editorial assistance and Donna Reynolds and Carol Oborn from the Department of

\section{REFERENCES}

Ahmad SA, Liu W, Jung YD, Fan F, Wilson M, Reinmuth N, Shaheen RM, Bucana CD, Ellis LM (2001) The effects of angiopoietin-1 and -2 on tumor growth and angiogenesis in human colon cancer. Cancer Res 61: 12551259

Asahara T, Chen D, Takahashi T, Fujikawa K, Kearney M, Magner M, Yancopoulos GD, Isner JM (1998) Tie2 receptor ligands, angiopoietin-1 and angiopoietin-2, modulate VEGF-induced postnatal neovascularization. Circ Res 83: $233-240$

Chae JK, Kim I, Lim ST, Chung MJ, Kim WH, Kim HG, Ko JK, Koh GY (2000) Coadministration of angiopoietin-1 and vascular endothelial growth factor enhances collateral vascularization. Arterioscler Thromb Vasc Biol 20: $2573-2578$

Dumont DJ, Gradwohl G, Fong GH, Puri MC, Gertsenstein M, Auerbach A, Breitman ML (1994) Dominant-negative and targeted null mutations in the endothelial receptor tyrosine kinase, tek, reveal a critical role in vasculogenesis of the embryo. Genes Dev 8: 1897-1909

Dvorak HF, Nagy JA, Feng D, Brown LF, Dvorak AM (1999) Vascular permeability factor/vascular endothelial growth factor and the significance of microvascular hyperpermeability in angiogenesis. Curr Top Microbiol Immunol 237: $97-132$

Dvorak HF (2000) VPF/VEGF and the angiogenic response. Semin Perinatol 24: $75-78$

Ellis LM, Liu W, Wilson M (1996) Down-regulation of vascular endothelial growth factor in human colon carcinoma cell lines by antisense transfection decreases endothelial cell proliferation. Surgery 120: 871-878

Fujikawa K, de Aos ScherpenseelI, Jain SK, Presman E, Christensen RA, Varticovski L (1999) Role of PI 3-kinase in angiopoietin-1-mediated migration and attachment-dependent survival of endothelial cells. Exp Cell Res 253: $663-672$

Gamble JR, Drew J, Trezise L, Underwood A, Parsons M, Kasminkas L, Rudge J, Yancopoulos G, Vadas MA (2000) Angiopoietin-1 is an antipermeability and anti-inflammatory agent in vitro and targets cell junctions. Circ Res 87: 603-607

Hansbury MJ, Nicosia RF, Zhu WH, Holmes SJ, Winkler JD (2001) Production and characterization of a Tie2 agonist monoclonal antibody. Angiogenesis 4: $29-36$

Hayes AJ, Huang WQ, Mallah J, Yang D, Lippman ME, Li LY (1999) Angiopoietin-1 and its receptor Tie-2 participate in the regulation of capillarylike tubule formation and survival of endothelial cells. Microvasc Res 58: $224-237$

Hayes AJ, Huang WQ, Yu J, Maisonpierre PC, Liu A, Kern FG, Lippman ME, McLeskey SW, Li LY (2000) Expression and function of angiopoietin-1 in breast cancer. Br J Cancer 83: 1154-1160

Holash J, Maisonpierre PC, Compton D, Boland P, Alexander CR, Zagzag D, Yancopoulos GD, Wiegand SJ (1999) Vessel cooption, regression, and growth in tumors mediated by angiopoietins and VEGF. Science 284: $1994-1998$

Kwak HJ, So JN, Lee SJ, Kim I, Koh GY (1999) Angiopoietin-1 is an apoptosis survival factor for endothelial cells. FEBS Lett 448: 249-253

Maisonpierre PC, Suri C, Jones PF, Bartunkova S, Wiegand SJ, Radziejewski C, Compton D, McClain J, Aldrich TH, Papadopoulos N, Daly TJ, Davis S, Sato TN, Yancopoulos GD (1997) Angiopoietin-2, a natural antagonist for Tie2 that disrupts in vivo angiogenesis. Science 277: 55-60
Cancer Biology for technical assistance. These studies were supported in part by NIH grant T-3209599 (to SA Ahmad and AA Parikh), NIH grant U54 CA90810-01 (to LM Ellis), a grant from The George and Barbara Bush Foundation for Innovative Cancer Research (to LM Ellis), and NIH Cancer Center Support Grant CA16672.

Morikawa K, Walker SM, Jessup JM, Fidler IJ (1988) In vivo selection of highly metastatic cells from surgical specimens of different primary human colon carcinomas implanted into nude mice. Cancer Res 48: 1943-1948

Papapetropoulos A, Garcia-Cardena G, Dengler TJ, Maisonpierre PC, Yancopoulos GD, Sessa WC (1999) Direct actions of angiopoietin-1 on human endothelium: evidence for network stabilization, cell survival, and interaction with other angiogenic growth factors. Lab Invest 79: 213-223

Peters KG (1998) Vascular endothelial growth factor and the angiopoietins: working together to build a better blood vessel. Circ Res 83: $342-343$

Ray PS, Estrada-Hernandez T, Sasaki H, Zhu L, Maulik N (2000) Early effects of hypoxia/reoxygenation on VEGF, ang-1, ang- 2 and their receptors in the rat myocardium: implications for myocardial angiogenesis. Mol Cell Biochem 213: $145-153$

Sato TN, Tozawa Y, Deutsch U, Wolburg-Buchholz K, Fujiwara Y, GendronMaguire M, Gridley T, Wolburg H, Risau W, Qin Y (1995) Distinct roles of the receptor tyrosine kinases Tie-1 and Tie-2 in blood vessel formation. Nature 376: 70 - 74

Shaheen RM, Ahmad SA, Liu W, Reinmuth N, Jung YD, Tseng WW, Drazan KE, Bucana CD, Hicklin DJ, Ellis LM (2001) Inhibited growth of colon cancer carcinomatosis by antibodies to vascular endothelial and epidermal growth factor receptors. Br J Cancer 85: 584-589

Shim WS, Teh M, Mack PO, Ge R (2001) Inhibition of angiopoietin-1 expression in tumor cells by an antisense RNA approach inhibited xenograft tumor growth in immunodeficient mice. Int J Cancer 94: 6-15

Suri C, Jones PF, Patan S, Bartunkova S, Maisonpierre PC, Davis S, Sato TN, Yancopoulos GD (1996) Requisite role of angiopoietin-1, a ligand for the TIE2 receptor, during embryonic angiogenesis. Cell 87: 1171-1180

Suri C, McClain J, Thurston G, McDonald DM, Zhou H, Oldmixon EH, Sato TN, Yancopoulos GD (1998) Increased vascularization in mice overexpressing angiopoietin-1. Science 282: $468-471$

Takahashi Y, Bucana CD, Cleary KR, Ellis LM (1998) p53, vessel count, and vascular endothelial growth factor expression in human colon cancer. Int $J$ Cancer 79: $34-38$

Takahashi Y, Kitadai Y, Bucana CD, Cleary KR, Ellis LM (1995) Expression of vascular endothelial growth factor and its receptor, KDR, correlates with vascularity, metastasis, and proliferation of human colon cancer. Cancer Res 55: 3964-3968

Thurston G, Rudge JS, Ioffe E, Zhou H, Ross L, Croll SD, Glazer N, Holash J, McDonald DM, Yancopoulos GD (2000) Angiopoietin-1 protects the adult vasculature against plasma leakage. Nat Med 6: 460-463

Thurston G, Suri C, Smith K, McClain J, Sato TN, Yancopoulos GD, McDonald DM (1999) Leakage-resistant blood vessels in mice transgenically overexpressing angiopoietin-1. Science 286: $2511-2514$

Tian S, Hayes AJ, Metheny-Barlow LJ, Li LY (2002) Stabilization of breast cancer xenograft tumour neovasculature by angiopoietin-1. Br J Cancer 86: $645-651$

United Kingdom Co-ordinating Committee on Cancer Research, (UKCCCR) (1998) Guidelines for the Welfare of Animals in Experimental Neoplasia (Second Edition). Br J Cancer 77: 1-10

Zebrowski BK, Yano S, Liu W, Shaheen RM, Hicklin DJ, Putnam Jr JB, Ellis LM (1999) Vascular endothelial growth factor levels and induction of permeability in malignant pleural effusions. Clin Cancer Res 5: 3364-3368 